\title{
A NEW GEOMETRIC SETTING FOR CLASSICAL FIELD THEORIES
}

\author{
M. DE LEÓN \\ Instituto de Matemáticas y Física Fundamental \\ Consejo Superior de Investigaciones Cientificas \\ Serrano 123, 28006 Madrid, Spain \\ E-mail:mdeleon@imaff.cfmac.csic.es \\ J. C. MARRERO \\ Departamento de Matemática Fundamental \\ Facultad de Matemáticas, Universidad de La Laguna \\ La Laguna, Tenerife, Canary Islands, Spain \\ E-mail: jcmarrer@ull.es \\ D. MARTÍN DE DIEGO \\ Instituto de Matemáticas y Física Fundamental \\ Consejo Superior de Investigaciones Científicas \\ Serrano 123, 28006 Madrid, Spain \\ E-mail:d.martin@imaff.cfmac.csic.es
}

\begin{abstract}
A new geometrical setting for classical field theories is introduced. This description is strongly inspired by the one due to Skinner and Rusk for singular lagrangian systems. For a singular field theory a constraint algorithm is developed that gives a final constraint submanifold where a well-defined dynamics exists. The main advantage of this algorithm is that the second order condition is automatically included.
\end{abstract}

1. Introduction. The search of a convenient setting for classical field theories has been an strong motivation for geometers and physicists in the last forty years. In the end of the sixties the so-called multisymplectic formalism was developed, which is a natural extension of the symplectic framework for mechanics.

2000 Mathematics Subject Classification: Primary 70S05; Secondary 58A20.

This work has been partially supported by grants BFM2001-2272 and BFM2000-0808.

The paper is in final form and no version of it will be published elsewhere. 
The multisymplectic approach was developed by the Polish school led by W. Tulczyjew (see [3] for more details), and independently by P. L. García and A. Pérez-Rendón [12, 13], and Goldschmidt and Sternberg [14]. This approach leads to a geometric definition of multisymplectic form in $[17,18]$, and more recently in $[4,5]$ where a careful study of these structures is developed (see also [27, 28] for previous results, and [2, 25, 29, 30] for recent developments).

There are two different ways to present the evolution equations in a geometric form. One uses the notion of Ehresmann connections [23, 24] which is widely employed along the present paper. The other one uses the notion of multivector field (see $[8,9,10,11]$ ). Of course, both are equivalent, and permit to develop a convenient constraint algorithm when we are dealing with singular lagrangians.

Alternative geometric approaches based on the so-called $n$-symplectic geometry (see [20] for a recent survey), and polysymplectic geometry (see [31, 32]) are also available.

The aim of the present paper is to give a new geometric setting, based on that developed by Skinner and Rusk [33, 34]. In order to treat with singular lagrangian systems, Skinner and Rusk have constructed a hamiltonian system on the Withney sum $T^{*} Q \oplus T Q$ over the configuration manifold $Q$. The advantage of their approach lies in the fact that the second order condition of the dynamics is automatically satisfied. This does not happen in the Gotay and Nester formulation, where the second order condition problem has to be considered after the implementation of the constraint algorithm (see $[15,16,21])$.

Here, we start with a lagrangian function defined on $Z$, where $\pi_{X Z}: Z \rightarrow X$ is the 1-jet prolongation of a fibration $\pi_{X Y}: Y \rightarrow X$. We consider the fibration $\pi_{X W_{0}}: W_{0} \rightarrow X$, where $W_{0}=\Lambda_{2}^{n} Y \times_{Y} Z$ is the fibered product. On $W_{0}$ we construct a multisymplectic form by pulling back the canonical multisymplectic form on $\Lambda_{2}^{n} Y$, and define a convenient hamiltonian. The solutions of the field equations are viewed as integral sections of Ehresmann connections in the fibration $\pi_{X Z}: Z \rightarrow X$. The resulting algorithm is compared with the ones developed in the lagrangian and hamiltonian settings. The scheme is applied to an example, the bosonic string. The case of time-dependent mechanics is recovered as a particular case. The paper also contains three appendices exhibiting some notions and properties of Ehresmann connections.

2. Lagrangian formalism. A classical field theory consists of a fibration $\pi_{X Y}: Y \rightarrow$ $X$ (that is, $\pi_{X Y}$ is a surjective submersion) over an orientable $n$-dimensional manifold $X$ and an $n$-form $\Lambda$ (the lagrangian form) defined on the 1-jet prolongation $\pi_{X Z}: J^{1} \pi_{X Y} \rightarrow$ $X$ along the projection $\pi_{X Y}$. We will use the notation $Z=J^{1} \pi_{X Y}$. In addition, if $\eta$ is a fixed volume form on $X$ we have $\Lambda=L \eta$, where $L$ is a function on $Z$. An additional fiber bundle $\pi_{Y Z}: Z \rightarrow Y$ is also obtained. Here $X$ represents the space-time manifold, and the fields are viewed as sections of $\pi_{X Y}$. (See $[3,17,18,19,31,32]$ ).

Definition 2.1. A lagrangian $L: Z \rightarrow \mathbb{R}$ is said to be regular if the hessian matrix

$$
\left(\frac{\partial^{2} L}{\partial z_{\mu}^{i} \partial z_{\nu}^{j}}\right)
$$

is regular. Otherwise, $L$ is said to be singular. 
Along this paper we will choose fibered coordinates $\left(x^{\mu}, y^{i}, z_{\mu}^{i}\right)$ on $Z$ such that $\eta=$ $d^{n} x=d x^{1} \wedge \ldots \wedge d x^{n}$. Here $\mu$ runs from 1 to $n$, and $i$ runs from 1 to $m$, so that $Y$ has dimension $n+m$. A useful notation will be $d^{n-1} x^{\mu}=i_{\frac{\partial}{\partial x^{\mu}}} \eta$.

The volume form $\eta$ permits to construct a tensor field of type $(1, n)$ on $Z$ :

$$
S_{\eta}=\left(d y^{i}-z_{\mu}^{i} d x^{\mu}\right) \wedge d^{n-1} x^{\nu} \otimes \frac{\partial}{\partial z_{\nu}^{i}} .
$$

Next, the Poincaré-Cartan $n$-form and $(n+1)$-form are defined as follows:

$$
\Theta_{L}=\Lambda+S_{\eta}^{*}(d L), \quad \Omega_{L}=-d \Theta_{L},
$$

where $S_{\eta}^{*}$ is the adjoint operator of $S_{\eta}$. In coordinates, we have

$$
\begin{aligned}
& \Theta_{L}=\left(L-z_{\mu}^{i} \frac{\partial L}{\partial z_{\mu}^{i}}\right) d^{n} x+\frac{\partial L}{\partial z_{\mu}^{i}} d y^{i} \wedge d^{n-1} x^{\mu} \\
& \Omega_{L}=-d\left(L-z_{\mu}^{i} \frac{\partial L}{\partial z_{\mu}^{i}}\right) \wedge d^{n} x-d\left(\frac{\partial L}{\partial z_{\mu}^{i}}\right) \wedge d y^{i} \wedge d^{n-1} x^{\mu} .
\end{aligned}
$$

An extremal of $L$ is a section $\phi$ of $\pi_{X Y}$ such that, for any vector field $\xi_{Z}$ on $Z$,

$$
\left(j^{1} \phi\right)^{*}\left(i_{\xi_{Z}} \Omega_{L}\right)=0
$$

where $j^{1} \phi$ is the first jet prolongation of $\phi$.

As is well-known, $\phi$ is an extremal of $L$ if and only if it satisfies the Euler-Lagrange equations:

$$
\left(j^{1} \phi\right)^{*}\left(\frac{\partial L}{\partial y^{i}}-\frac{d}{d x^{\mu}}\left(\frac{\partial L}{\partial z_{\mu}^{i}}\right)\right)=0, \quad 1 \leq i \leq n .
$$

We can consider a more general kind of solutions, those sections $\psi$ of the fiber bundle $\pi_{X Z}: Z \rightarrow X$ such that

$$
\psi^{*}\left(i_{\xi_{Z}} \Omega_{L}\right)=0
$$

for any vector field $\xi_{Z}$ on $Z$. Equation (3) is referred as the de Donder equations.

Looking at (3) we have an alternative characterization. Let $\Gamma$ be an Ehresmann connection in $\pi_{X Z}: Z \rightarrow X$, with horizontal projector $\mathbf{h}$. Consider the equation

$$
i_{\mathbf{h}} \Omega_{L}=(n-1) \Omega_{L} .
$$

The horizontal sections (if they exist) of $\Gamma$ are just the solutions of the de Donder problem.

Indeed, if

$$
\mathbf{h}\left(\frac{\partial}{\partial x^{\mu}}\right)=\frac{\partial}{\partial x^{\mu}}+\Gamma_{\mu}^{i} \frac{\partial}{\partial y^{i}}+\Gamma_{\nu \mu}^{i} \frac{\partial}{\partial z_{\nu}^{i}}
$$

then a direct computation shows that equation (4) holds if and only if

$$
\begin{array}{r}
\left(\Gamma_{\nu}^{j}-z_{\nu}^{j}\right)\left(\frac{\partial^{2} L}{\partial z_{\mu}^{i} \partial z_{\nu}^{j}}\right)=0 \\
\frac{\partial L}{\partial y^{i}}-\frac{\partial^{2} L}{\partial x^{\mu} \partial z_{\mu}^{i}}-\Gamma_{\mu}^{j} \frac{\partial^{2} L}{\partial y^{j} \partial z_{\mu}^{i}}-\Gamma_{\mu \nu}^{j} \frac{\partial^{2} L}{\partial z_{\nu}^{j} \partial z_{\mu}^{i}}+\left(\Gamma_{\nu}^{j}-z_{\nu}^{j}\right) \frac{\partial^{2} L}{\partial y^{i} \partial z_{\nu}^{j}}=0
\end{array}
$$

(see $[23])$. 
If the lagrangian $L$ is regular, then Eq. (5) implies that $\Gamma_{\mu}^{i}=z_{\mu}^{i}$ and therefore (6) becomes

$$
\frac{\partial L}{\partial y^{i}}-\frac{\partial^{2} L}{\partial x^{\mu} \partial z_{\mu}^{i}}-z_{\mu}^{j} \frac{\partial^{2} L}{\partial y^{j} \partial z_{\mu}^{i}}-\Gamma_{\mu \nu}^{j} \frac{\partial^{2} L}{\partial z_{\nu}^{j} \partial z_{\mu}^{i}}=0 .
$$

Now, if $\tau\left(x^{\mu}\right)=\left(x^{\mu}, \tau^{i}(x), \tau_{\mu}^{i}(x)\right)$ is an integral section of $\Gamma$ we would have

$$
z_{\mu}^{i}=\frac{\partial \tau^{i}}{\partial x^{\mu}} \quad \Gamma_{\mu \nu}^{i}=\frac{\partial \tau_{\mu}^{i}}{\partial x^{\nu}}
$$

which proves that Eq. (7) is nothing but the Euler-Lagrange equations for $L$.

If the lagrangian $L$ is regular, then every solution $\psi$ of the de Donder equations (3) is automatically a 1-jet prolongation, say $\psi=j^{1} \phi$ and the section $\phi$ of $\pi_{X Y}$ is a solution of equations (1).

In terms of Ehresmann connections, if $L$ is regular, then any solution $\Gamma$ of equations (4) is semi-holonomic (see Appendix B).

3. Hamiltonian formulation. Let $\Lambda_{r}^{n} Y, 1 \leq r \leq m$, be the subbundle of the bundle $\Lambda^{n} Y$ of $n$-forms on $Y$ consisting of those $n$-forms which vanish when $r$ of their arguments are vertical. We have a chain of vector bundles over $Y$ :

$$
0 \subset \Lambda_{1}^{n} Y \subset \Lambda_{2}^{n} Y \subset \cdots \subset \Lambda^{n} Y .
$$

The elements of $\Lambda_{1}^{n} Y$ (resp. $\Lambda_{2}^{n} Y$ ) are locally expressed as $p(x, y) d^{n} x$ (resp. $p d^{n} x+$ $\left.p_{i}^{\mu} d y^{i} \wedge d^{n-1} x^{\mu}\right)$. Thus, we introduce local coordinates $\left(x^{\mu}, y^{i}, p\right)$ on the manifold $\Lambda_{1}^{n} Y$, and $\left(x^{\mu}, y^{i}, p, p_{i}^{\mu}\right)$ on $\Lambda_{2}^{n} Y$.

The manifold $\Lambda^{n} Y$ carries a canonical $n$-form, $\Theta_{0}$, which is defined as follows:

$$
\Theta_{0}(\omega)\left(\xi_{1}, \xi_{2}, \ldots, \xi_{n}\right)=\omega(\nu(\omega))\left(\nu_{*}\left(\xi_{1}\right), \nu_{*}\left(\xi_{2}\right), \ldots, \nu_{*}\left(\xi_{n}\right)\right)
$$

where $\omega \in \Lambda^{n} Y, \xi_{i} \in T_{\omega}\left(\Lambda^{n} Y\right)$, and $\nu: \Lambda^{n} Y \rightarrow Y$ is the canonical projection.

This form $\Theta_{0}$ induces an $n$-form $\Theta_{r}$ on $\Lambda_{r}^{n} Y$, for each $r, 1 \leq r \leq m$.

The closed $(n+1)$-forms $\Omega_{r}=-d \Theta_{r}$ (and of course, $\left.\Omega_{0}=-d \Theta_{0}\right)$ are examples of the so-called multisymplectic forms according the following definition.

Definition 3.1. A multisymplectic form on a manifold $M$ is a closed $k$-form $\Omega$ on $M$ such that the linear mapping $v \in T_{x} M \rightarrow i_{v} \Omega \in \Lambda^{k-1} T_{x}^{*} M$ is injective for all $x \in M$. The manifold $M$ equipped with a multisymplectic form $\Omega$ will be called a multisymplectic manifold, usually denoted by the pair $(M, \Omega)$. Two multisymplectic manifolds $(M, \Omega)$ and $(\bar{M}, \bar{\Omega})$ will be said multisymplectomorphic if there exists a diffeomorphism $\phi: M \rightarrow \bar{M}$ preserving the multisymplectic forms, say $\phi^{*} \bar{\Omega}=\Omega ; \phi$ will be called a multisymplectomorphism.

REMARK 3.2. It will be useful to write the local expressions of the canonical multisymplectic forms on $\Lambda_{2}^{n} Y$ :

$$
\Theta_{2}=p d^{n} x+p_{i}^{\mu} d y^{i} \wedge d^{n-1} x^{\mu}, \quad \Omega_{2}=-d p \wedge d^{n} x-d p_{i}^{\mu} \wedge d y^{i} \wedge d^{n-1} x^{\mu} .
$$

A direct computation shows the following.

Proposition 3.3. Assume that $n \geq 2$. Then, a lagrangian $L$ is regular if and only if the pair $\left(Z, \Omega_{L}\right)$ is a multisymplectic manifold. 
Since $\Lambda_{1}^{n} Y$ is a vector subbundle of $\Lambda_{2}^{n} Y$ we can construct the quotient vector bundle $\Lambda_{2}^{n} Y / \Lambda_{1}^{n} Y$ which will be denoted by $Z^{*}$. The projection $\Lambda_{2}^{n} Y \rightarrow Z^{*}$ will be denoted by $\lambda$. We also have a fibration $\pi_{X Z^{*}}: Z^{*} \rightarrow X$.

In this context, a hamiltonian $h$ is a section of $\lambda$. Using this hamiltonian we define an $n$-form $\Theta_{h}$ on $Z^{*}$ by pulling back the canonical $n$-form $\Theta_{2}$, i.e. $\Theta_{h}=h^{*} \Theta_{2}$. We put $\Omega_{h}=-d \Theta_{h}$ so that $\Omega_{h}=h^{*} \Omega_{2}$.

A section $\sigma$ of $\pi_{X Z^{*}}: Z^{*} \rightarrow X$ is said to satisfy the Hamilton equations for a given hamiltonian $h$ if

$$
\sigma^{*}\left(i_{\xi_{Z^{*}}} \Omega_{h}\right)=0
$$

for any vector fields $\xi_{Z^{*}}$ on $Z^{*}$.

In local coordinates $\left(x^{\mu}, y^{i}, p_{i}^{\mu}\right)$ for $Z^{*}$, the section $h$ may be represented by a local function $H$ :

$$
p=-H\left(x^{\mu}, y^{i}, p_{i}^{\mu}\right)
$$

then

$$
\Theta_{h}=-H d^{n} x+p_{i}^{\mu} d y^{i} \wedge d^{n-1} x^{\mu}, \quad \Omega_{h}=d H \wedge d^{n} x-d p_{i}^{\mu} \wedge d y^{i} \wedge d^{n-1} x^{\mu},
$$

and the Hamilton equations for a section $\sigma$ become:

$$
\frac{\partial y^{i}}{\partial x^{\mu}}=\frac{\partial H}{\partial p_{i}^{\mu}}, \quad \frac{\partial p_{i}^{\mu}}{\partial x^{\mu}}=-\frac{\partial H}{\partial y^{i}} .
$$

As in the preceding section, we can consider a connection $\widetilde{\Gamma}$ in $\pi_{X Z^{*}}: Z^{*} \rightarrow X$, with horizontal projector $\widetilde{\mathbf{h}}$. An intrinsic version of equations (10) is then the following:

$$
i_{\widetilde{\mathbf{h}}} \Omega_{h}=(n-1) \Omega_{h} .
$$

Indeed, if $\widetilde{\Gamma}$ is flat, then its integral sections are solutions of the Hamilton equations.

REMARK 3.4. If $n \geq 2$ then, from (9), it follows that $\Omega_{h}$ is a multisymplectic form on $Z^{*}$.

4. The Legendre transformation. Let $L$ be a lagrangian function. We define a fiber preserving map

$$
\operatorname{leg}_{L}: Z \rightarrow \Lambda_{2}^{n} Y
$$

as follows:

$$
\operatorname{leg}_{L}\left(j_{x}^{1} \phi\right)\left(X_{1}, \ldots, X_{n}\right)=\left(\Theta_{L}\right)_{j_{x}^{1} \phi}\left(\tilde{X}_{1}, \ldots, \tilde{X}_{n}\right)
$$

for all $j_{x}^{1} \phi \in Z$ and $X_{i} \in T_{\phi(x)} Y$, where $\tilde{X}_{i} \in T_{j_{x}^{1} \phi} Z$ are such that $\left(\pi_{Y Z}\right)_{*}\left(\tilde{X}_{i}\right)=X_{i}$.

In local coordinates, we have

$$
\operatorname{leg}_{L}\left(x^{\mu}, y^{i}, z_{\mu}^{i}\right)=\left(x^{\mu}, y^{i}, p=L-z_{\mu}^{i} \frac{\partial L}{\partial z_{\mu}^{i}}, p_{i}^{\mu}=\frac{\partial L}{\partial z_{\mu}^{i}}\right) .
$$

The Legendre transformation $\operatorname{Leg}_{L}: Z \rightarrow Z^{*}$ is defined as the composition $\operatorname{Leg}_{L}=$ $\lambda \circ \operatorname{leg}_{L}$, and it is locally expressed as

$$
\operatorname{Leg}_{L}\left(x^{\mu}, y^{i}, z_{\mu}^{i}\right)=\left(x^{\mu}, y^{i}, \frac{\partial L}{\partial z_{\mu}^{i}}\right) \text {. }
$$

From the definitions, we deduce that $\left(\operatorname{leg}_{L}\right)^{*} \Theta_{2}=\Theta_{L}$ and $\left(\operatorname{leg}_{L}\right)^{*} \Omega_{2}=\Omega_{L}$. 
Proposition 4.1. The lagrangian $L$ is regular if and only if the Legendre transformation $\operatorname{Leg}_{L}: Z \rightarrow Z^{*}$ is a local diffeomorphism.

The Legendre transformation permits to connect the lagrangian and hamiltonian descriptions as follows.

Assume that the lagrangian $L$ is hyper-regular, that is, $\operatorname{Leg}_{L}: Z \rightarrow Z^{*}$ is a global diffeomorphism. We define a hamiltonian section $h: Z^{*} \rightarrow \Lambda_{2}^{n} Y$ by setting

$$
h=\operatorname{leg}_{L} \circ\left(\operatorname{Leg}_{L}\right)^{-1} \text {. }
$$

Then, from (12) it follows that

$$
\operatorname{Leg}_{L}^{*} \Theta_{h}=\Theta_{L}, \quad \operatorname{Leg}_{L}^{*} \Omega_{h}=\Omega_{L}
$$

Therefore, the solutions of equations (3) and (8) are $\operatorname{Leg}_{L}$-related. In terms of connections, the solutions of equations (4) and (11) are also $\operatorname{Leg}_{L}$-related.

If the lagrangian is regular, the equivalence is only at local level. More precisely, if $n \geq 2$, we have that $\operatorname{Leg}_{L}$ is a local multisymplectomorphism between the multisymplectic manifolds $\left(Z, \Omega_{L}\right)$ and $\left(Z^{*}, \Omega_{h}\right)$.

For singular lagrangians, a constraint algorithm was developed in [23] (see Section 6).

5. A new geometric setting. Consider the fibered product $W_{0}=\Lambda_{2}^{n} Y \times_{Y} Z$ with canonical projections $\operatorname{pr}_{1}: W_{0} \rightarrow \Lambda_{2}^{n} Y$ and $\operatorname{pr}_{2}: W_{0} \rightarrow Z$. We consider fibered coordinates $\left(x^{\mu}, y^{i}, p, p_{i}^{\mu}, z_{\mu}^{i}\right)$ on $W_{0}$.

Define the $n$-form $\Theta=\operatorname{pr}_{1}^{*} \Theta_{2}$ and the $(n+1)$-form $\Omega=-d \Theta=\operatorname{pr}_{1}^{*} \Omega_{2}$.

We also define a function $\Phi: W_{0} \rightarrow \mathbb{R}$ as follows. Take an element $\left(\omega_{\phi(x)}, j_{x}^{1} \phi\right) \in W_{0}$, then $\Phi\left(\left(\omega_{\phi(x)}, j_{x}^{1} \phi\right)\right)=a(x)$, where

$$
\phi^{*}\left(\omega_{\phi(x)}\right)=a(x) \eta(x) .
$$

Locally, we have

$$
\Phi\left(x^{\mu}, y^{i}, p, p_{i}^{\mu}, z_{\mu}^{i}\right)=p+p_{i}^{\mu} z_{\mu}^{i} .
$$

Define also the function $H_{0}: W_{0} \rightarrow \mathbb{R}$ by setting

$$
H_{0}=\Phi-\operatorname{pr}_{2}^{*} L .
$$

The function $H_{0}$ locally reads as

$$
H_{0}\left(x^{\mu}, y^{i}, p, p_{i}^{\mu}, z_{\mu}^{i}\right)=p+p_{i}^{\mu} z_{\mu}^{i}-L\left(x^{\mu}, y^{i}, z_{\mu}^{i}\right) .
$$

Put

$$
\Omega_{H_{0}}=\Omega+d H_{0} \wedge \eta
$$

In local coordinates we have

$$
\Omega_{H_{0}}=-d p \wedge d^{n} x-d p_{i}^{\mu} \wedge d y^{i} \wedge d^{n-1} x^{\mu}+d H_{0} \wedge d^{n} x .
$$

Let $\bar{\Gamma}$ be an Ehresmann connection in the fibered bundle $\pi_{X W_{0}}: W_{0} \rightarrow X$, with horizontal projector $\overline{\mathbf{h}}$.

We search for a solution of the equation:

$$
i_{\overline{\mathbf{h}}} \Omega_{H_{0}}=(n-1) \Omega_{H_{0}} .
$$


Define

$$
\begin{aligned}
W_{1}= & \left\{u \in W_{0} / \exists \overline{\mathbf{h}}_{u}: T_{u} W_{0} \rightarrow T_{u} W_{0} \quad \text { linear such that } \overline{\mathbf{h}}_{u}^{2}=\overline{\mathbf{h}}_{u},\right. \\
& \left.\operatorname{ker} \overline{\mathbf{h}}_{u}=\left(V \pi_{X W_{0}}\right)_{u}, i_{\overline{\mathbf{h}}_{u}} \Omega_{H_{0}}(u)=(n-1) \Omega_{H_{0}}(u)\right\} .
\end{aligned}
$$

Suppose that the local expression of $\overline{\mathbf{h}}$ is

$$
\begin{aligned}
\overline{\mathbf{h}}\left(\frac{\partial}{\partial x^{\mu}}\right) & =\frac{\partial}{\partial x^{\mu}}+A_{\mu}^{i} \frac{\partial}{\partial y^{i}}+B_{\mu} \frac{\partial}{\partial p}+C_{\mu i}^{\nu} \frac{\partial}{\partial p_{i}^{\nu}}+D_{\mu \nu}^{i} \frac{\partial}{\partial z_{\nu}^{i}} \\
\overline{\mathbf{h}}\left(\frac{\partial}{\partial y^{i}}\right) & =0, \quad \overline{\mathbf{h}}\left(\frac{\partial}{\partial p}\right)=0, \\
\overline{\mathbf{h}}\left(\frac{\partial}{\partial p_{i}^{\mu}}\right) & =0, \quad \overline{\mathbf{h}}\left(\frac{\partial}{\partial z_{\mu}^{i}}\right)=0 .
\end{aligned}
$$

We then obtain

$$
\begin{aligned}
i_{\overline{\mathbf{h}}} \Omega_{H_{0}}= & i_{\overline{\mathbf{h}}}\left(-d p \wedge d^{n} x-d p_{i}^{\mu} \wedge d y^{i} \wedge d^{n-1} x^{\mu}+d H_{0} \wedge d^{n} x\right) \\
= & (n-1) \Omega_{H_{0}}+\left(C_{\mu i}^{\mu}-\frac{\partial L}{\partial y^{i}}\right) d y^{i} \wedge d^{n} x \\
& +\left(z_{\mu}^{i}-A_{\mu}^{i}\right) d p_{i}^{\mu} \wedge d^{n} x+\left(p_{i}^{\mu}-\frac{\partial L}{\partial z_{\mu}^{i}}\right) d z_{\mu}^{i} \wedge d^{n} x
\end{aligned}
$$

Therefore, the submanifold $W_{1}$ of $W_{0}$ is determined by the vanishing of the constraints:

$$
p_{i}^{\mu}-\frac{\partial L}{\partial z_{\mu}^{i}}=0,
$$

and the components of the connection $\overline{\mathbf{h}}$ would satisfy the following relations:

$$
\begin{gathered}
A_{\mu}^{i}=z_{\mu}^{i}, \\
C_{\mu i}^{\mu}=\frac{\partial L}{\partial y^{i}} .
\end{gathered}
$$

From the definition of $W_{1}$ we know that for each point $u \in W_{1}$ there exists a "horizontal projector" $\overline{\mathbf{h}}_{u}: T_{u} W_{0} \rightarrow T_{u} W_{0}$ satisfying equation (13). However, we cannot ensure that such $\overline{\mathbf{h}}_{u}$ for each $u \in W_{1}$ will take values in $T_{u} W_{1}$.

But notice that the condition $\overline{\mathbf{h}}_{u}\left(T_{u} W_{0}\right) \subset T_{u} W_{1}, \forall u \in W_{1}$ is equivalent to having

$$
\overline{\mathbf{h}}\left(\frac{\partial}{\partial x^{\mu}}\right)\left(p_{j}^{\kappa}-\frac{\partial L}{\partial z_{\kappa}^{j}}\right)=0
$$

or, equivalently,

$$
C_{\mu j}^{\kappa}=\frac{\partial^{2} L}{\partial z_{\kappa}^{j} \partial x^{\mu}}+z_{\mu}^{i} \frac{\partial^{2} L}{\partial z_{\kappa}^{j} \partial y^{i}}+D_{\mu \nu}^{i} \frac{\partial^{2} L}{\partial z_{\kappa}^{j} \partial z_{\nu}^{i}} .
$$

We remark that if the lagrangian $L$ is regular, then equations (16) have solutions $D$ 's for a particular choice of $C$ 's satisfying equations (15). Of course, we can take arbitrary values for the $B$ 's. A global solution is obtained using partitions of unity.

In such a case, we obtain by restriction a connection $\bar{\Gamma}$ in the fiber bundle $\pi_{X W_{1}}$ : $W_{1} \rightarrow X$, which is a solution of equation (13) when it is restricted to $W_{1}$ (in fact, we have a family of such solutions). Assume that $\bar{\Gamma}$ is flat, and $\bar{\psi}$ is a horizontal section of $\bar{\Gamma}$. First 
of all, notice that $\bar{\psi}$ takes values in $W_{1}$ which implies that $\psi=\operatorname{pr}_{2} \circ \bar{\psi}$ is a jet prolongation. Let us explain better this assertion. If $\bar{\psi}\left(x^{\mu}\right)=\left(x^{\mu}, y^{i}(x), p(x), p_{i}^{\mu}(x), z_{\mu}^{i}(x)\right)$ then we have

$$
z_{\mu}^{i}(x)=\frac{\partial y^{i}}{\partial x^{\mu}}
$$

Since

$$
D_{\mu \nu}^{i}=\frac{\partial z_{\nu}^{i}}{\partial x^{\mu}}
$$

we deduce that along $\psi$ we have

$$
\frac{\partial L}{\partial y^{j}}-\frac{\partial^{2} L}{\partial z_{\mu}^{j} \partial x^{\mu}}-\frac{\partial y^{i}}{\partial x^{\mu}} \frac{\partial^{2} L}{\partial z_{\mu}^{j} \partial y^{i}}-\frac{\partial z_{\nu}^{i}}{\partial x^{\mu}} \frac{\partial^{2} L}{\partial z_{\mu}^{j} \partial z_{\nu}^{i}}=0
$$

that is,

$$
\frac{\partial L}{\partial y^{j}}-\frac{d}{d x^{\mu}}\left(\frac{\partial L}{\partial z_{\mu}^{j}}\right)=0
$$

which are the Euler-Lagrange equations for $L$.

Up to now, we have no assigned any meaning to the coordinate $p$. Consider the submanifold $\bar{W}_{1}$ of $W_{1}$ defined by the equation $H_{0}=0$. In other words, $\bar{W}_{1}$ is locally characterized by the equation

$$
p=-\left(p_{i}^{\mu} z_{\mu}^{i}-L\right)
$$

which defines a local energy.

We can ask when a solution exists on $\bar{W}_{1}$. Indeed, it is possible to construct a family of connections in the fiber bundle $\pi_{X \bar{W}_{1}}: \bar{W}_{1} \rightarrow X$ which solve equation (13) as follows.

We have to choose coefficients $B_{\mu}, C_{\mu i}^{\nu}$, and $D_{\mu \nu}^{i}$ verifying (15) and (16), and in addition,

$$
\bar{h}\left(\frac{\partial}{\partial x^{\mu}}\right)\left(H_{0}\right)=0
$$

A direct computation shows that (17) is equivalent to the following local conditions:

$$
B_{\mu}+C_{\mu i}^{\nu} z_{\nu}^{i}=\frac{\partial L}{\partial x^{\mu}}+z_{\mu}^{i} \frac{\partial L}{\partial y^{i}}
$$

Now, if we choose appropriate values for $C_{\mu i}^{\nu}$ satisfying (15) and (16), then we can take the values for $B_{\mu}$ given by equation (18). A global solution is finally obtained using partitions of the unity.

Denote by $\Omega_{\bar{W}_{1}}$ the restriction of $\Omega_{H_{0}}$ to $\bar{W}_{1}$.

Proposition 5.1. If $n \geq 2$ and the Lagrangian $L$ is regular then $\Omega_{\bar{W}_{1}}$ is a multisymplectic form.

Proof. The result follows from a direct computation taking into account that on $W_{1}$ we have

$$
p_{i}^{\mu}=\frac{\partial L}{\partial z_{\mu}^{i}}
$$

and that the hessian matrix

$$
\left(\frac{\partial^{2} L}{\partial z_{\mu}^{i} \partial z_{\nu}^{j}}\right)
$$

is regular. 
Next, we shall relate the above construction with the preceding ones in the lagrangian and the hamiltonian settings.

First of all, the following results are quite obvious:

- The submanifold $\bar{W}_{1}$ is diffeomorphic to $Z$.

- If $n \geq 2$ and $L$ is (hyper)regular, then the multisymplectic manifolds $\left(\bar{W}_{1}, \Omega_{\bar{W}_{1}}\right)$, $\left(Z, \Omega_{L}\right)$ and $\left(Z^{*}, \Omega_{h}\right)$ are (globally) locally multisymplectomorphic. Indeed, the corresponding multisymplectomorphisms are the following ones:

$$
\left(\operatorname{pr}_{2}\right)_{\left.\right|_{\bar{W}_{1}}}: \bar{W}_{1} \rightarrow Z, \quad \operatorname{Leg}_{L}: Z \rightarrow Z^{*}, \quad \operatorname{Leg}_{L} \circ\left(\mathrm{pr}_{2}\right)_{\left.\right|_{\bar{W}_{1}}}: \bar{W}_{1} \rightarrow Z^{*}
$$

(Note that $\left.\lambda \circ\left(\mathrm{pr}_{1}\right)_{\left.\right|_{\bar{W}_{1}}}=\operatorname{Leg}_{L} \circ\left(\mathrm{pr}_{2}\right)_{\left.\right|_{\bar{W}_{1}}}\right)$.

- As a consequence, one can choose connections $\mathbf{h}, \tilde{\mathbf{h}}$ and $\overline{\mathbf{h}}$ in the fibrations $\pi_{X Z}$ : $Z \rightarrow X, \pi_{X Z^{*}}: Z^{*} \rightarrow X$, and $\pi_{X \bar{W}_{1}}: \bar{W}_{1} \rightarrow X$, respectively, such that they are solutions of equations (4), (11) and (13), respectively, and, in addition, they are related by the above multisymplectomorphisms.

The following diagram summarizes the above discussion:

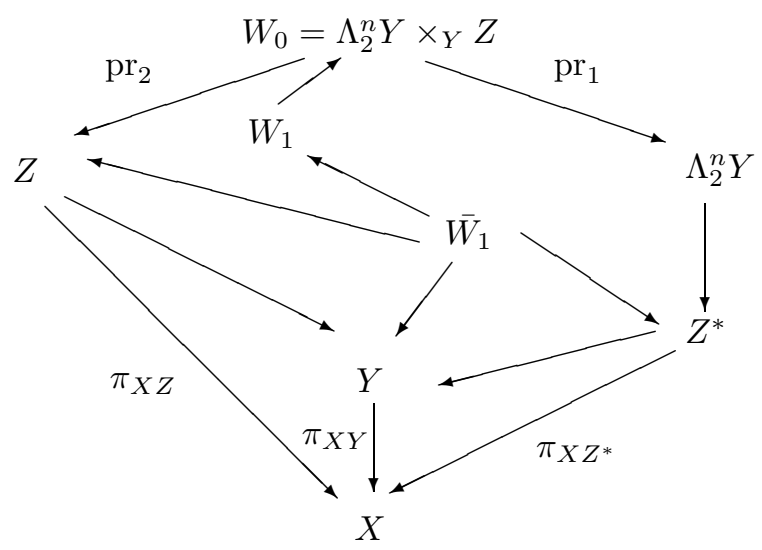

6. Singular lagrangians. For a singular lagrangian $L$, we usually have to go further in the constraint algorithm. Therefore, we will consider a subset $\bar{W}_{2}$ defined in order to satisfy the tangency condition:

$$
\begin{aligned}
\bar{W}_{2}= & \left\{u \in \bar{W}_{1} / \exists \overline{\mathbf{h}}_{u}: T_{u} W_{0} \rightarrow T_{u} \bar{W}_{1} \quad \text { linear such that } \overline{\mathbf{h}}_{u}^{2}=\overline{\mathbf{h}}_{u},\right. \\
& \left.\operatorname{ker} \overline{\mathbf{h}}_{u}=\left(V \pi_{X W_{0}}\right)_{u}, i_{\overline{\mathbf{h}}_{u}} \Omega_{H_{0}}(u)=(n-1) \Omega_{H_{0}}(u)\right\} .
\end{aligned}
$$

Assume that $\bar{W}_{2}$ is a submanifold of $\bar{W}_{1}$. If $\overline{\mathbf{h}}_{u}\left(T_{u} W_{0}\right)$ is not contained in $T_{u} \bar{W}_{2}$, we go to the next step, and so on.

At the end, and if the system has solutions, we will find a final constraint submanifold $\bar{W}_{f}$, fibered over $X$ (or over some open subset of $X$ ) (see Appendix C) and a connection $\bar{\Gamma}_{f}$ in this fibration such that $\bar{\Gamma}_{f}$ is a solution of equation (13) restricted to $\bar{W}_{f}$.

Similar constraint algorithms can be developed using equations (4) and (11). Our purpose in the following is to relate these three algorithms. 
Indeed, we can consider the subset

$$
\begin{aligned}
Z_{2}= & \left\{z \in Z / \exists \mathbf{h}_{z}: T_{z} Z \rightarrow T_{z} Z \text { linear such that } \mathbf{h}_{z}^{2}=\mathbf{h}_{z},\right. \\
& \left.\operatorname{ker} \mathbf{h}_{z}=\left(V \pi_{X Z}\right)_{z}, i_{\mathbf{h}_{z}} \Omega_{L}(z)=(n-1) \Omega_{L}(z)\right\} .
\end{aligned}
$$

If $Z_{2}$ is a submanifold, then there are solutions but we have to include the tangency condition, and consider a new step:

$$
\begin{aligned}
Z_{3}= & \left\{z \in Z_{2} / \exists \mathbf{h}_{z}: T_{z} Z \rightarrow T_{z} Z_{2} \quad \text { linear such that } \mathbf{h}_{z}^{2}=\mathbf{h}_{z},\right. \\
& \left.\operatorname{ker} \mathbf{h}_{z}=\left(V \pi_{X Z}\right)_{z}, i_{\mathbf{h}_{z}} \Omega_{L}(z)=(n-1) \Omega_{L}(z)\right\} .
\end{aligned}
$$

If $Z_{3}$ is a submanifold of $Z_{2}$, but $\mathbf{h}_{z}\left(T_{z} Z\right)$ is not contained in $T_{z} Z_{3}$, we go to the next step, and so on. Finally, we will obtain (in the favorable cases) a final constraint submanifold $Z_{f}$ and a connection in the fibration $\pi_{X Z}: Z \rightarrow X$ along the submanifold $Z_{f}$ (in fact, a family of connections) with horizontal projector $\mathbf{h}$ which is a solution of equation (4).

There is an additional problem, since our connection would be a solution of the de Donder problem, but not a solution of the Euler-Lagrange equations. This problem is solved by constructing a submanifold of $Z_{f}$ where such a solution exists (see $[23,24]$ and below for more details).

To develop a hamiltonian counterpart, we need some weak regularity of the lagrangian $L$.

Definition 6. 1. A lagrangian $L: Z \rightarrow \mathbb{R}$ is said to be almost regular if $\operatorname{leg}_{L}(Z)=\tilde{Z}$ is a submanifold of $\Lambda_{2}^{n} Y$, and $\operatorname{leg}_{L}: Z \rightarrow \tilde{Z}$ is a submersion with connected fibers.

If $L$ is almost regular, one has:

- $\tilde{Z}_{1}=\operatorname{Leg}_{L}(Z)$ is a submanifold of $Z^{*}$, and in addition, a fibration over $X$.

- The restriction $\lambda_{1}: \tilde{Z} \rightarrow \tilde{Z}_{1}$ of $\lambda$ is a diffeomorphism.

- The mapping $\operatorname{Leg}_{1}: Z \rightarrow \tilde{Z}_{1}$ is a submersion with connected fibers.

Define a mapping $h_{1}=\left(\lambda_{1}\right)^{-1}: \tilde{Z}_{1} \rightarrow \tilde{Z}$, and a $(n+1)$-form $\tilde{\Omega}_{1}$ on $\tilde{Z}_{1}$ by $\tilde{\Omega}_{1}=$ $h_{1}^{*}\left(\left(\Omega_{2}\right)_{\left.\right|_{\tilde{Z}}}\right)$. Obviously, we have $\operatorname{Leg}_{1}^{*} \tilde{\Omega}_{1}=\Omega_{L}$.

The hamiltonian description is now based on the equation

$$
i_{\tilde{\mathbf{h}}} \tilde{\Omega}_{1}=(n-1) \tilde{\Omega}_{1}
$$

where $\tilde{\mathbf{h}}$ is a connection in the fibration $\pi_{X \tilde{Z}_{1}}: \tilde{Z}_{1} \rightarrow X$.

Proceeding as above, we construct a constraint algorithm as follows.

First, we define

$$
\begin{aligned}
\tilde{Z}_{2}= & \left\{\tilde{z} \in \tilde{Z}_{1} / \exists \tilde{\mathbf{h}}_{\tilde{z}}: T_{\tilde{z}} \tilde{Z}_{1} \rightarrow T_{\tilde{z}} \tilde{Z}_{1} \quad \text { linear such that } \tilde{\mathbf{h}}_{\tilde{z}}^{2}=\tilde{\mathbf{h}}_{\tilde{z}},\right. \\
& \left.\operatorname{ker} \tilde{\mathbf{h}}_{\tilde{z}}=\left(V \pi_{X \tilde{Z}_{1}}\right) \tilde{z}_{\tilde{z}}, i_{\tilde{\mathbf{h}}_{\tilde{z}}} \tilde{\Omega}_{1}(\tilde{z})=(n-1) \tilde{\Omega}_{1}(\tilde{z})\right\} .
\end{aligned}
$$

If $\tilde{Z}_{2}$ is a submanifold, then there are solutions but we have to include the tangency condition, and consider a new step:

$$
\begin{aligned}
\tilde{Z}_{3}= & \left\{\tilde{z} \in \tilde{Z}_{2} / \exists \tilde{\mathbf{h}}_{\tilde{z}}: T_{\tilde{z}} \tilde{Z}_{1} \rightarrow T_{\tilde{z}} \tilde{Z}_{2} \quad \text { linear such that } \tilde{\mathbf{h}}_{\tilde{z}}^{2}=\tilde{\mathbf{h}}_{\tilde{z}},\right. \\
& \left.\operatorname{ker} \tilde{\mathbf{h}}_{\tilde{z}}=\left(V \pi_{X \tilde{Z}_{1}}\right) \tilde{z}, i_{\tilde{\mathbf{h}}_{\tilde{z}}} \tilde{\Omega}_{1}(\tilde{z})=(n-1) \tilde{\Omega}_{1}(\tilde{z})\right\} .
\end{aligned}
$$

If $\tilde{Z}_{3}$ is a submanifold of $\tilde{Z}_{2}$, but $\tilde{\mathbf{h}}_{\tilde{z}}\left(T_{\tilde{z}} \tilde{Z}_{1}\right)$ is not contained in $T_{\tilde{z}} \tilde{Z}_{3}$, we go to the next step, and so on. Finally, we will obtain (in the favorable cases) a final constraint sub- 
manifold $\tilde{Z}_{f}$ and a connection in the fibration $\pi_{X \tilde{Z}_{1}}: \tilde{Z}_{1} \rightarrow X$ along the submanifold $\tilde{Z}_{f}$ (in fact, a family of connections) with horizontal projector $\tilde{\mathbf{h}}$ which is a solution of equation (11).

The important facts are the following:

- The mapping $\operatorname{Leg}_{1}: Z \rightarrow \tilde{Z}_{1}$ preserves the constraint algorithms, that is, we have $\operatorname{Leg}_{1}\left(Z_{r}\right)=\tilde{Z}_{r}$ for each integer $r \geq 2$.

- In consequence, both algorithms have the same behavior; in particular, if one of them stabilizes, the same happens with the other, and at the same step, so we have $\operatorname{Leg}_{1}\left(Z_{f}\right)=\tilde{Z}_{f}$.

- In the latter case, the restriction $\operatorname{Leg}_{f}: Z_{f} \rightarrow \tilde{Z}_{f}$ is a surjective submersion (that is, a fibration) and $\operatorname{Leg}_{f}^{-1}\left(\operatorname{Leg}_{f}(z)\right)=\operatorname{Leg}_{1}^{-1}\left(\operatorname{Leg}_{1}(z)\right)$, for all $z \in Z_{f}$.

Therefore, the lagrangian and hamiltonian sides can be compared through the fibration $\operatorname{Leg}_{f}: Z_{f} \rightarrow \tilde{Z}_{f}$. Indeed, if we have a connection in the fibration $\pi_{X Z}: Z \rightarrow X$ along the submanifold $Z_{f}$ with horizontal projector $\mathbf{h}$ which is a solution of equation (4) (the de Donder equation) and, in addition, the connection is projectable via $\operatorname{Leg}_{f}$ to a connection in the fibration $\pi_{X \tilde{Z}}: \tilde{Z} \rightarrow X$ along the submanifold $\tilde{Z}_{f}$, then the horizontal projector of the projected connection is a solution of equation (11) (the Hamilton equations). Conversely, given a connection in the fibration $\pi_{X \tilde{Z}}: \tilde{Z} \rightarrow X$ along the submanifold $\tilde{Z}_{f}$, with horizontal projector $\tilde{\mathbf{h}}$ which is a solution of equation (11), then every connection in the fibration $\pi_{X Z}: Z \rightarrow X$ along the submanifold $Z_{f}$ that projects onto $\tilde{\mathbf{h}}$ is a solution of the de Donder equation (4).

Assume that $L$ is almost regular and construct the above algorithms. Take a $L_{e} g_{f^{-}}$ projectable connection $\Gamma$ in the fibration $\pi_{X Z}: Z \rightarrow X$ along the submanifold $Z_{f}$ with horizontal projector $\mathbf{h}$ which is a solution of equation (4), and denote by $\tilde{\Gamma}$ its projection. As we have shown, the horizontal projector $\tilde{\mathbf{h}}$ is a solution of equation (11).

In general, $\Gamma$ is not semi-holonomic, that is, $S_{\eta}(\mathbf{h}, \ldots, \mathbf{h}) \not \equiv 0$ along $Z_{f}$. However, we can define a section $\beta$ of the fibration $\operatorname{Leg}_{L}: Z_{f} \rightarrow \tilde{Z}_{f}$ such that

$$
\left(S_{\eta}(\mathbf{h}, \ldots, \mathbf{h})\right)_{\left.\right|_{\beta\left(\tilde{z}_{f}\right)}}=0 .
$$

The construction of $\beta$ is based on the following interpretation of the elements of $Z$.

Take $z \in Z$, that is, $z$ is a 1-jet of a section $\phi$ of the fibration $\pi_{X Y}: Y \rightarrow X$. Since $\mathbf{H}_{\phi(x)}=T \phi(x)\left(T_{x} X\right)$ is a horizontal subspace of $T_{\phi(x)} Y$, for every $x \in X$ (in fact, in the domain of $\phi$ ) we can identify $z$ with this horizontal subspace, which in local coordinates means that if $z=\left(x^{\mu}, y^{i}, z_{\mu}^{i}\right)$, then $\mathbf{H}_{\phi(x)}$ is spanned by the tangent vectors $\partial / \partial x^{\mu}+z_{\mu}^{i} \partial / \partial y^{i}$

With the above notations and the obvious identifications, we define

$$
\beta(\tilde{z})=T \pi_{Y Z}\left(\mathbf{h}\left(T_{z_{0}} Z\right)\right),
$$

where $z_{0} \in Z_{f}$ is an arbitrary point projecting onto $\tilde{z}$ through the projection $\operatorname{Leg}_{f}$ : $Z_{f} \rightarrow \tilde{Z}_{f}$

We have:

- $\beta(\tilde{z})$ is independent of the choice of $z_{0}$. This is a consequence of the following two facts: $\mathbf{h}$ projects onto $\tilde{\mathbf{h}}$, and $\pi_{X Z^{*}} \circ \operatorname{Leg}_{f}=\pi_{X Z}$. 
- The point $\beta(\tilde{z})$ belongs to $Z_{f}$. Indeed, consider the local vector field

$$
U=\left(\Gamma_{\mu}^{i}-z_{\mu}^{i}\right) \frac{\partial}{\partial z_{\mu}^{i}},
$$

where $\Gamma_{\mu}^{i}$ are the Christoffel components of $\Gamma$, that is,

$$
\mathbf{h}\left(\frac{\partial}{\partial x^{\mu}}\right)=\frac{\partial}{\partial x^{\mu}}+\Gamma_{\mu}^{i} \frac{\partial}{\partial y^{i}}+\Gamma_{\mu \nu}^{i} \frac{\partial}{\partial z_{\nu}^{i}} .
$$

Since $\Gamma$ is $L e g_{f}$-projectable, $\Gamma_{\mu}^{i}$ is constant along the fiber over $\tilde{z}$.

From (5) and (12), we deduce that $U$ is a vertical vector field with respect to the fibration $\operatorname{Leg}_{f}: Z_{f} \rightarrow \tilde{Z}_{f}$, and in consequence it is tangent to the fiber over $\tilde{z}$. Consider the curve

$$
\alpha(t)=\left(\left(x^{\mu}\right)_{0},\left(y^{i}\right)_{0},\left(\Gamma_{\mu}^{i}\right)_{0}-\exp (-t)\left(\left(\Gamma_{\mu}^{i}\right)_{0}-\left(z_{\mu}^{i}\right)_{0}\right)\right)
$$

where $\left(\left(x^{\mu}\right)_{0},\left(y^{i}\right)_{0},\left(z_{\mu}^{i}\right)_{0}\right)$ are the coordinates of $z_{0}$, and $\left(\Gamma_{\mu}^{i}\right)_{0}$ are the values of $\Gamma_{\mu}^{i}$ at the point $z_{0}$ (in fact, along the whole fiber). $\alpha(t)$ is an integral curve of $U$ passing through $z_{0}$ and totally contained in the fiber over $\tilde{z}$. Thus, the limit point $\lim _{t \rightarrow+\infty} \alpha(t)$ is in this fiber, and a direct computation shows that $\lim _{t \rightarrow+\infty} \alpha(t)=$ $\beta(\tilde{z})$.

- Now, it is obvious that $\Gamma$ is semiholonomic at the point $\beta(\tilde{z})$.

Since $\beta$ is a section, we deduce that $\beta\left(\tilde{Z}_{f}\right)$ is a submanifold of $Z_{f}$ and hence of $Z$. In addition, $\left(\operatorname{Leg}_{f}\right)_{\left.\right|_{\beta\left(\tilde{Z}_{f}\right)}}: \beta\left(\tilde{Z}_{f}\right) \rightarrow \tilde{Z}_{f}$ is a diffeomorphism.

Next, we define a connection $\Gamma_{s}$ in the fibration $\pi_{X Z}: Z \rightarrow X$ along $\beta\left(\tilde{Z}_{f}\right)$ as follows. Its horizontal projector is given by

$$
\left(\mathbf{h}_{s}\right)_{z}: T_{z} Z \rightarrow T_{z} \beta\left(\tilde{Z}_{f}\right), \quad\left(\mathbf{h}_{s}\right)_{z}=\left(T\left(\operatorname{Leg}_{f}\right)_{\left.\right|_{\beta\left(\tilde{Z}_{f}\right)}}(z)\right)^{-1} \circ \tilde{\mathbf{h}}_{z} \circ T \operatorname{Leg}_{f}(z),
$$

for all $z \in \beta\left(\tilde{Z}_{f}\right)$, where $z=\beta(\tilde{z})$. A straightforward computation shows that $\Gamma_{s}$ is a solution of (4) and, in addition, is transported onto $\tilde{\Gamma}$ via the diffeomorphism $\left(\operatorname{Leg}_{L}\right)_{\left.\right|_{\beta\left(\tilde{Z}_{f}\right)}}$ : $\beta\left(\tilde{Z}_{f}\right) \rightarrow \tilde{Z}_{f}$. Thus, since $\Gamma$ is semiholonomic along $\beta\left(\tilde{Z}_{f}\right)$, we deduce that $\Gamma_{s}$ is also semiholonomic along $\beta\left(\tilde{Z}_{f}\right)$.

Next, we will relate the above constructions with the algorithm developed from equation (13).

To do that, we first develop an alternative constraint algorithm based on the following equation:

$$
i_{\hat{\mathbf{h}}} \Omega_{\bar{W}_{1}}=(n-1) \Omega_{\bar{W}_{1}},
$$

where $\Omega_{\bar{W}_{1}}$ is the restriction of $\Omega_{H_{0}}$ to $\bar{W}_{1}$, and $\hat{\mathbf{h}}$ is the horizontal projector of a connection $\hat{\Gamma}$ in the fibration $\pi_{X \bar{W}_{1}}=\left(\pi_{X W_{0}}\right)_{\left.\right|_{\bar{W}_{1}}}: \bar{W}_{1} \rightarrow X$.

The algorithm proceeds now as in the above cases, and it produces a chain of submanifolds (in the favorable cases). Indeed, we define

$$
\begin{aligned}
\hat{W}_{2}= & \left\{u \in \bar{W}_{1} / \exists \hat{\mathbf{h}}_{u}: T_{u} \bar{W}_{1} \rightarrow T_{u} \bar{W}_{1} \quad \text { linear such that } \hat{\mathbf{h}}_{u}^{2}=\hat{\mathbf{h}}_{u},\right. \\
& \left.\operatorname{ker} \hat{\mathbf{h}}_{u}=\left(V \pi_{X \bar{W}_{1}}\right)_{u}, i_{\hat{\mathbf{h}}_{u}} \Omega_{\bar{W}_{1}}(u)=(n-1) \Omega_{\bar{W}_{1}}(u)\right\} .
\end{aligned}
$$

If we assume that $\hat{W}_{2}$ is a submanifold of $\bar{W}_{1}$, since in general $\hat{\mathbf{h}}_{u}\left(T_{u} \bar{W}_{1}\right)$ is not contained in $T_{u} \hat{W}_{2}$, we go to the next step, and so on. 
At the end, and if the system has solutions, we will find a final constraint submanifold $\hat{W}_{f}$, fibered over $X$ (or over some open subset of $X$ ) (see Appendix C) and a connection $\hat{\Gamma}_{f}$ in this fibration such that $\hat{\Gamma}_{f}$ is a solution of equation (21) restricted to $\hat{W}_{f}$.

It should be noticed that $\bar{W}_{r} \subset \hat{W}_{r}$, for all integer $r \geq 2$. Indeed, any pointwise solution of equation (13) is a solution of equation (21). As a consequence, both algorithms have the same behavior.

This last algorithm can be compared with the lagrangian and hamiltonian ones. In fact, since

$$
\left(\tilde{\mathrm{pr}}_{2}\right)^{*} \Omega_{L}=\Omega_{\bar{W}_{1}}, \quad\left(\tilde{\mathrm{pr}}_{1}\right)^{*} \tilde{\Omega}_{1}=\Omega_{\bar{W}_{1}},
$$

where $\tilde{p r}_{1}=\lambda_{1} \circ\left(\operatorname{pr}_{1}\right)_{\left.\right|_{\bar{W}_{1}}}$ and $\tilde{p r}_{2}=\left(\mathrm{pr}_{2}\right)_{\left.\right|_{\bar{W}_{1}}}$, we have

$$
\tilde{\operatorname{pr}}_{1}\left(\hat{W}_{r}\right)=\tilde{Z}_{r}, \quad \tilde{\operatorname{pr}}_{2}\left(\hat{W}_{r}\right)=Z_{r}
$$

for all $r \geq 2$, and a fortiori we deduce that all the algorithms have the same behavior and

$$
\tilde{\operatorname{pr}}_{1}\left(\hat{W}_{f}\right)=\tilde{Z}_{f}, \quad \tilde{\operatorname{pr}}_{2}\left(\hat{W}_{f}\right)=Z_{f}
$$

Thus, the corresponding solutions can be related via the convenient projections. More precisely, we can construct a connection $\Gamma$ (resp. $\tilde{\Gamma}, \hat{\Gamma})$ in the fibration $\pi_{X Z}: Z \rightarrow X$ (resp. $\pi_{X \tilde{Z}_{1}}: \tilde{Z}_{1} \rightarrow X, \pi_{X \bar{W}_{1}}: \bar{W}_{1} \rightarrow X$ ) along the submanifold $Z_{f}$ (resp. $\tilde{Z}_{f}, \hat{W}_{f}$ ) such that they are related by the projections $L e g_{f}, \tilde{\mathrm{pr}}_{1}$ and $\tilde{\mathrm{pr}}_{2}$.

In addition, the connection $\Gamma$ can be chosen such that its restriction to $\bar{W}_{f}$ is a solution of equation (13). Making all these selections, and performing the construction of the section $\beta$ we conclude that $\beta\left(\tilde{Z}_{f}\right) \subset \bar{W}_{f}$.

The following diagram summarizes the above discussion:

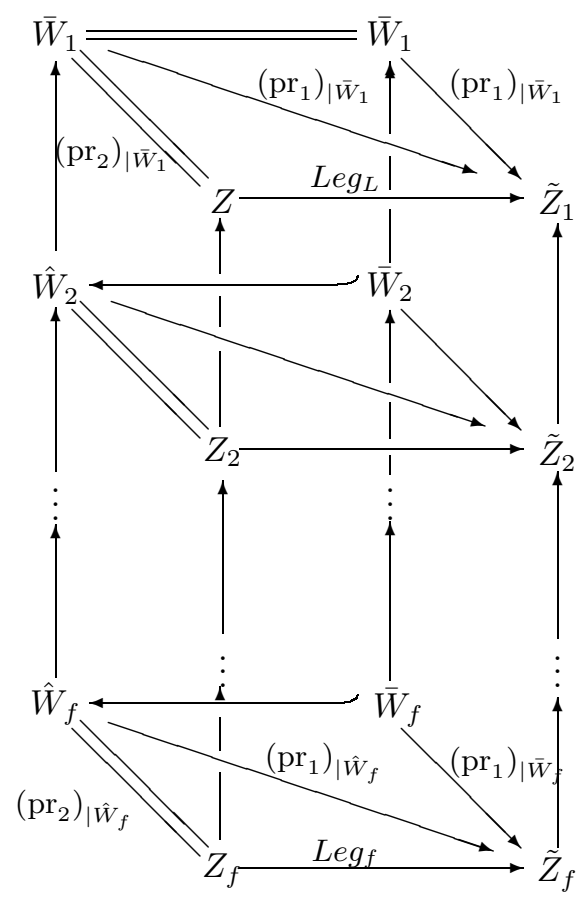


REMARK 6.2. According to Appendix C, one has that all the connections considered in this section define bona fide connections in the corresponding restricted fibrations

$$
\begin{aligned}
\pi_{X_{0} Z_{f}}: Z_{f} \rightarrow X_{0}, \\
\pi_{X_{0} \tilde{Z}_{f}}: \tilde{Z}_{f} \rightarrow X_{0}, \\
\pi_{X_{0} \bar{W}_{f}}: \bar{W}_{f} \rightarrow X_{0}, \\
\pi_{X_{0} \hat{W}_{f}}: \hat{W}_{f} \rightarrow X_{0},
\end{aligned}
$$

where $X_{0}$ is an open submanifold of $X$.

7. Example: The bosonic string (see $[1,17]$ ). Let $X$ be a 2-dimensional manifold, and $(B, g)$ a $d+1$-dimensional spacetime manifold endowed with a Lorentz metric $g$ of signature $(-,+, \ldots,+)$. A bosonic string is a map $\phi: X \rightarrow B$.

In the following, we will follow the Polyakov approach to classical bosonic string theory. Let $S_{2}^{1,1}(X)$ be the bundle over $X$ of symmetric 2-covariant tensors with signature $(-,+)$ or $(1,1)$. We take the vector bundle $\pi: Y=X \times B \times S_{2}^{1,1}(X) \rightarrow X$. Therefore, in this formulation, a field $\psi$ is a section $(\phi, h)$ of the vector bundle $Y=X \times B \times S_{2}^{1,1}(X) \rightarrow$ $X$, where $\phi: X \rightarrow Y$ is the bosonic string and $h$ is a Lorentz metric on $X$.

Lagrangian description. We have that $Z=J^{1}(X \times B) \times_{X} J^{1}\left(S_{2}^{1,1}(X)\right)$. Taking coordinates $\left(x^{\mu}\right),\left(y^{i}\right)$ and $\left(x^{\mu}, h_{\mu \eta}\right)$ on $X, B$ and $S_{2}^{1,1}(X)$ then the canonical local coordinates on $Z$ are $\left(x^{\mu}, y^{i}, h_{\eta \xi}, y_{\mu}^{i}, h_{\eta \xi \mu}\right)$. In this system of local coordinates, the Lagrangian density is given by

$$
\Lambda=-\frac{1}{2} \sqrt{-\operatorname{det}(h)} h^{\eta \xi} g_{i j} y_{\eta}^{i} y_{\xi}^{j} d^{2} x
$$

The Cartan 3-form is

$$
\begin{aligned}
\Omega_{L}= & d y^{i} \wedge d\left(-\sqrt{-\operatorname{det}(h)} h^{\eta \xi} g_{i j} y_{\xi}^{j}\right) \wedge d^{1} x^{\eta} \\
& -d\left(\frac{1}{2} \sqrt{-\operatorname{det}(h)} h^{\eta \xi} g_{i j} y_{\eta}^{i} y_{\xi}^{j}\right) \wedge d^{2} x \\
= & -\frac{1}{2}\left(\frac{\partial \sqrt{-\operatorname{det}(h)}}{\partial h_{\rho \sigma}} h^{\eta \xi} g_{i j} y_{\eta}^{i} y_{\xi}^{j}-\sqrt{-\operatorname{det}(h)} h^{\eta \rho} h^{\xi \sigma} g_{i j} y_{\eta}^{i} y_{\xi}^{j}\right) d h_{\rho \sigma} \wedge d^{2} x \\
& -\frac{1}{2} \sqrt{-\operatorname{det}(h)} h^{\eta \xi} \frac{\partial g_{i j}}{\partial y^{k}} y_{\eta}^{i} y_{\xi}^{j} d y^{k} \wedge d^{2} x-\sqrt{-\operatorname{det}(h)} h^{\eta \xi} g_{i j} y_{\eta}^{i} d y_{\xi}^{j} \wedge d^{2} x \\
& +\left(\frac{\partial \sqrt{-\operatorname{det}(h)}}{\partial h_{\rho \sigma}} h^{\eta \xi} g_{i j} y_{\xi}^{j}-\sqrt{-\operatorname{det}(h)} h^{\eta \rho} h^{\xi \sigma} g_{i j} y_{\xi}^{j}\right) d h_{\rho \sigma} \wedge d y^{i} \wedge d^{1} x^{\eta} \\
& +\sqrt{-\operatorname{det}(h)} h^{\eta \xi} \frac{\partial g_{i j}}{\partial y^{k}} y_{\xi}^{j} d y^{k} \wedge d y^{i} \wedge d^{1} x^{\eta} \\
& +\sqrt{-\operatorname{det}(h)} h^{\eta \xi} g_{i j} d y_{\xi}^{j} \wedge d y^{i} \wedge d^{1} x^{\eta} .
\end{aligned}
$$

If we solve the equation $i_{\mathbf{h}} \Omega_{L}=\Omega_{L}$, where

$$
\mathbf{h}=d x^{\mu} \otimes\left(\frac{\partial}{\partial x^{\mu}}+\Gamma_{\mu}^{i} \frac{\partial}{\partial y^{i}}+\gamma_{\eta \xi \mu} \frac{\partial}{\partial h_{\eta \xi}}+\Gamma_{\eta \mu}^{i} \frac{\partial}{\partial y_{\eta}^{i}}+\gamma_{\eta \xi \rho \mu} \frac{\partial}{\partial h_{\eta \xi \rho}}\right)
$$

we obtain that: 


$$
\begin{aligned}
\Gamma_{\mu}^{i}= & y_{\mu}^{i} \\
0= & \frac{1}{2} \sqrt{-\operatorname{det}(h)} h^{\eta \xi} \frac{\partial g_{i j}}{\partial y^{k}} y_{\eta}^{i} y_{\xi}^{j}-\sqrt{-\operatorname{det}(h)} h^{\eta \xi} \frac{\partial g_{k j}}{\partial y^{i}} y_{\eta}^{i} y_{\xi}^{j}-\sqrt{-\operatorname{det}(h)} h^{\eta \xi} g_{k j} \Gamma_{\xi \eta}^{j} \\
& -\left(\frac{\partial \sqrt{-\operatorname{det}(h)}}{\partial h_{\rho \sigma}} h^{\eta \xi} g_{k j} y_{\xi}^{j}-\sqrt{-\operatorname{det}(h)} h^{\eta \rho} h^{\xi \sigma} g_{k j} y_{\xi}^{j}\right) \gamma_{\rho \sigma \eta},
\end{aligned}
$$

and the constraints are given by the equations

$$
\frac{\partial}{\partial h_{\rho \theta}}\left(\sqrt{-\operatorname{det}(h)} h^{\eta \xi}\right) g_{i j} y_{\eta}^{i} y_{\xi}^{j}=0 .
$$

The previous equation corresponds to the following three constraints:

$$
\begin{gathered}
{\left[h^{\eta 0} h^{\xi 0}\left(h_{01}^{2}-h_{00} h_{11}\right)+\frac{1}{2} h^{\eta \xi} h_{11}\right] g_{i j} y_{\eta}^{i} y_{\xi}^{j}=0,} \\
{\left[h^{\eta 1} h^{\xi 1}\left(h_{01}^{2}-h_{00} h_{11}\right)+\frac{1}{2} h^{\eta \xi} h_{00}\right] g_{i j} y_{\eta}^{i} y_{\xi}^{j}=0,} \\
{\left[h^{\eta 0} h^{\xi 1}\left(h_{01}^{2}-h_{00} h_{11}\right)-h^{\eta \xi} h_{01}\right] g_{i j} y_{\eta}^{i} y_{\xi}^{j}=0,}
\end{gathered}
$$

which determine $Z_{2}$.

Hamiltonian description. The Legendre transformation is given by

$$
\operatorname{Leg}_{L}\left(x^{\mu}, y^{i}, h_{\eta \xi}, y_{\mu}^{i}, h_{\eta \xi \mu}\right)=\left(x^{\mu}, y^{i}, h_{\eta \xi},-\sqrt{-\operatorname{det}(h)} h^{\mu \eta} g_{i j} y_{\eta}^{j}, 0\right) .
$$

Therefore, the Lagrangian $L$ is almost-regular and, moreover, $\tilde{Z}_{1}=\operatorname{Im} \operatorname{Leg}_{L} \cong \tilde{Z}=$ $\operatorname{leg}_{L}(Z) \cong J^{1}(X \times B) \times_{X} S_{2}^{1,1}(X)$. Take now coordinates $\left(x^{\mu}, y^{i}, h_{\eta \xi}, p_{i}^{\mu}\right)$ on $\tilde{Z}_{1}$ and consider the mapping $h_{1}: \tilde{Z}_{1} \rightarrow \tilde{Z}$ given by

$$
h_{1}\left(x^{\mu}, y^{i}, h_{\eta \xi}, p_{i}^{\mu}\right)=\left(x^{\mu}, y^{i}, h_{\eta \xi}, p=\frac{1}{2 \sqrt{-\operatorname{det}(h)}} h_{\eta \xi} g^{i j} p_{\eta}^{i} p_{\xi}^{j}, p_{i}^{\mu}\right) .
$$

Then, we have

$$
\tilde{\Omega}_{1}=-d\left(\frac{1}{2 \sqrt{-\operatorname{det}(h)}} h_{\eta \xi} g^{i j} p_{i}^{\eta} p_{j}^{\xi}\right) \wedge d^{2} x+d y^{i} \wedge d p_{i}^{\mu} \wedge d^{1} x^{\mu}
$$

and the Hamilton equations are given by $i_{\tilde{\mathbf{h}}} \tilde{\Omega}_{1}=\tilde{\Omega}_{1}$,

$$
\tilde{\mathbf{h}}=d x^{\mu} \otimes\left(\frac{\partial}{\partial x^{\mu}}+\tilde{\Gamma}_{\mu}^{i} \frac{\partial}{\partial y^{i}}+\tilde{\gamma}_{\eta \xi \mu} \frac{\partial}{\partial h_{\eta \xi}}+\tilde{\Gamma}_{i \mu}^{\eta} \frac{\partial}{\partial p_{i}^{\eta}}\right) .
$$

Solving the above equation, we obtain

$$
\begin{aligned}
\tilde{\Gamma}_{\mu}^{i} & =-\frac{1}{\sqrt{-\operatorname{det}(h)}} h_{\eta \mu} g^{i j} p_{j}^{\eta}, \\
\tilde{\Gamma}_{i \mu}^{\mu} & =\frac{1}{2 \sqrt{-\operatorname{det}(h)}} h_{\eta \xi} \frac{\partial g^{i j}}{\partial y^{k}} p_{\eta}^{i} p_{\xi}^{j},
\end{aligned}
$$

and the secondary constraints

$$
\frac{g^{i j}}{\sqrt{-\operatorname{det}(h)}}\left(\frac{1}{2 \operatorname{det}(h)} \frac{\partial \operatorname{det}(h)}{\partial h_{\rho \sigma}} h_{\eta \xi} p_{i}^{\eta} p_{j}^{\xi}-p_{i}^{\rho} p_{j}^{\sigma}\right)=0
$$

determining $\tilde{Z}_{2}$. 
The new geometrical setting. We have that $W_{0}=\Lambda_{2}^{2} Y \times_{Y} Z$ with fibered coordinates

$$
\left(x^{\mu}, y^{i}, h_{\eta \xi}, p, p_{i}^{\mu}, q^{\eta \xi \mu}, y_{\mu}^{i}, h_{\eta \xi \mu}\right) \text {. }
$$

Therefore,

$$
\begin{aligned}
H_{0} & =p+p_{i}^{\mu} y_{\mu}^{i}+q^{\eta \xi \mu} h_{\eta \xi \mu}+\frac{1}{2} \sqrt{-\operatorname{det}(h)} h^{\eta \xi} g_{i j} y_{\eta}^{i} y_{\xi}^{j} \\
\Omega_{H_{0}} & =-d p \wedge d^{2} x-d p_{i}^{\mu} \wedge d y^{i} \wedge d^{1} x^{\mu}-d q^{\eta \xi \mu} \wedge d h_{\eta \xi} \wedge d^{1} x^{\mu}+d H_{0} \wedge d^{2} x .
\end{aligned}
$$

Consider now an Ehresmann connection in the fibered manifold $\pi_{X W_{0}}: W_{0} \rightarrow X$ with horizontal projector:

$$
\begin{aligned}
\overline{\mathbf{h}}= & d x^{\mu} \otimes\left(\frac{\partial}{\partial x^{\mu}}+A_{\mu}^{i} \frac{\partial}{\partial y^{i}}+A_{\eta \xi \mu} \frac{\partial}{\partial h_{\eta \xi}}+B_{\mu} \frac{\partial}{\partial p}+C_{\mu i}^{\eta} \frac{\partial}{\partial p_{i}^{\eta}}+C_{\eta \xi \sigma \mu} \frac{\partial}{\partial q^{\eta \xi \sigma}}\right. \\
& \left.+D_{\eta \mu}^{i} \frac{\partial}{\partial y_{\eta}^{i}}+D^{\eta \xi \sigma \mu} \frac{\partial}{\partial h_{\eta \xi \sigma}}\right)
\end{aligned}
$$

Solving $i_{\overline{\mathbf{h}}} \Omega_{H_{0}}=\Omega_{H_{0}}$ we obtain that the submanifold $W_{1}$ is determined by the constraints:

$$
\begin{aligned}
p_{i}^{\mu} & =-\sqrt{-\operatorname{det}(h)} h^{\mu \eta} g_{i j} y_{\eta}^{j}, \\
q^{\eta \xi \mu} & =0 .
\end{aligned}
$$

Let $\bar{W}_{1}$ be the submanifold of $W_{1}$ defined by the equation $H_{0}=0$, that is,

$$
p=\frac{1}{2} \sqrt{-\operatorname{det}(h)} h^{\eta \xi} g_{i j} y_{\eta}^{i} y_{\xi}^{j} .
$$

$\bar{W}_{1}$ is locally defined by coordinates $\left(x^{\mu}, y^{i}, h_{\eta \xi}, y_{\mu}^{i}, h_{\eta \xi \mu}\right)$.

In these coordinates, the solutions of equation (21) are exactly the same as the ones obtained in the lagrangian setting, and $\hat{W}_{2}$, as a submanifold of $W_{0}$, is determined by the following constraints:

$$
\begin{aligned}
p_{i}^{\mu}+\sqrt{-\operatorname{det}(h)} h^{\mu \eta} g_{i j} y_{\eta}^{j} & =0, \\
q^{\eta \xi \mu} & =0, \\
p-\frac{1}{2} \sqrt{-\operatorname{det}(h)} h^{\eta \xi} g_{i j} y_{\eta}^{i} y_{\xi}^{j} & =0, \\
\frac{\partial \sqrt{-\operatorname{det}(h)}}{\partial h_{\rho \sigma}} h^{\eta \xi} g_{i j} y_{\eta}^{i} y_{\xi}^{j}-\sqrt{-\operatorname{det}(h)} h^{\eta \rho} h^{\xi \sigma} g_{i j} y_{\eta}^{i} y_{\xi}^{j} & =0 .
\end{aligned}
$$

It is easy to show that $\bar{W}_{2}=\hat{W}_{2}$ and the solutions of equation (13) are the solutions of equation (21) which, in addition, are semiholonomic.

8. Time-dependent mechanics. The jet bundle description of time-dependent mechanical systems takes $X=\mathbb{R}$ and $\eta=d t$, where $t$ is the usual coordinate on $\mathbb{R}$ (see, for instance, [22]).

If $L: Z \rightarrow \mathbb{R}$ is a lagrangian function, $\Omega_{L}$ is the Poincaré-Cartan 2-form on $Z$ and $\eta_{Z}$ is the 1 -form on $Z$ defined by $\eta_{Z}=\left(\pi_{\mathbb{R} Z}\right)^{*}(\eta)$, then the de Donder equation (4) can be written as 


$$
i_{\xi_{Z}} \Omega_{L}=0, \quad i_{\xi_{Z}} \eta_{Z}=1
$$

where $\xi_{Z}$ is a vector field on $Z$. The integral curves of $\xi_{Z}$ are the solutions of the de Donder problem.

The lagrangian function $L$ is regular if and only if the pair $\left(\Omega_{L}, \eta_{Z}\right)$ is a cosymplectic structure on $Z$. We recall that a cosymplectic structure on a manifold $M$ of odd dimension $2 n+1$ is a pair which consists of a closed 2-form $\Omega$ and a closed 1-form $\eta$ such that $\eta \wedge \Omega^{n}$ is a volume form.

If $L$ is regular then there exists a unique vector field $\xi_{Z}$ which satisfies (22). In fact, $\xi_{Z}$ is the Reeb vector field of the cosymplectic structure $\left(\Omega_{L}, \eta_{Z}\right)$ and it is a second order differential equation, that is, $S_{d t} \xi_{Z}=0$. The trajectories of $\xi_{Z}$ are the solutions of the Euler-Lagrange equations.

On the other hand, in this case, $\Lambda_{2}^{1} Y$ is the cotangent bundle $T^{*} Y$ of the manifold $Y$ and $\Omega_{0}$ is the canonical symplectic structure of $T^{*} Y$. Moreover, if $h: Z^{*} \rightarrow \Lambda_{2}^{1} Y=T^{*} Y$ is a hamiltonian and $\eta_{Z^{*}}=\left(\pi_{\mathbb{R} Z^{*}}\right)^{*}(d t)$, then: i) the pair $\left(\Omega_{h}, \eta_{Z^{*}}\right)$ is a cosymplectic structure on $Z^{*}$ and ii) the solutions of the Hamilton equations are just the integral curves of the Reeb vector field $\xi_{h}$ of the cosymplectic structure $\left(\Omega_{h}, \eta_{Z^{*}}\right)$.

It should be noticed that if the lagrangian $L$ is regular and $\eta_{\bar{W}_{1}}=$ $\left(\pi_{\mathbb{R} \bar{W}_{1}}\right)^{*}(d t)$, we have that the pair $\left(\Omega_{\bar{W}_{1}}, \eta_{\bar{W}_{1}}\right)$ is again a cosymplectic structure on $\bar{W}_{1}$ and there exists a unique solution of equation (13) restricted to $\bar{W}_{1}$, namely, the Reeb vector field of the cosymplectic structure $\left(\Omega_{\bar{W}_{1}}, \eta_{\bar{W}_{1}}\right)$. Furthermore, if $L$ is (regular) hyperregular then the maps $\left(\mathrm{pr}_{2}\right)_{\mid \bar{W}_{1}}: \bar{W}_{1} \rightarrow Z, \operatorname{Leg}_{L}: Z \rightarrow Z^{*}$ and $\operatorname{Leg}_{L} \circ\left(\operatorname{pr}_{2}\right)_{\mid \bar{W}_{1}}: \bar{W}_{1} \rightarrow Z^{*}$ are (local) cosymplectomorphisms between the cosymplectic manifolds $\left(\bar{W}_{1}, \Omega_{\bar{W}_{1}}, \eta_{\bar{W}_{1}}\right)$, $\left(Z, \Omega_{L}, \eta_{Z}\right)$ and $\left(Z^{*}, \Omega_{h}, \eta_{Z^{*}}\right)$, where $h=\operatorname{leg}_{L} \circ\left(\operatorname{Leg}_{L}\right)^{-1}$. Thus, the Reeb vector fields $\xi_{\bar{W}_{1}}, \xi_{Z}$ and $\xi_{Z^{*}}$ are related by the above cosymplectomorphisms.

When the lagrangian $L$ is singular, we can develop the two algorithms using equations (13) and (21) and we obtain the corresponding constraint submanifolds

$$
\begin{aligned}
& \bar{W}_{i}=\left\{u \in \bar{W}_{i-1} / \exists \xi \in T_{u} \bar{W}_{i-1}, i_{\xi} \Omega_{H_{0}}(u)=0, \eta_{\bar{W}_{1}}(\xi)=1\right\}, \\
& \hat{W}_{i}=\left\{u \in \hat{W}_{i-1} / \exists \xi \in T_{u} \hat{W}_{i-1}, i_{\xi} \Omega_{\bar{W}_{1}}(u)=0, \eta_{\bar{W}_{1}}(\xi)=1\right\},
\end{aligned}
$$

for all $i \geq 2$, with $\bar{W}_{1}=\hat{W}_{1}$ (see Section 6$)$.

If $L$ is almost regular, then we have that

$$
\begin{aligned}
& \bar{W}_{i} \subset \hat{W}_{i}, \\
& \tilde{\operatorname{pr}}_{1}\left(\hat{W}_{i}\right)=\tilde{Z}_{i}=\left\{\tilde{z} \in \tilde{Z}_{i-1} / \exists \tilde{\xi} \in T_{\tilde{z}} \tilde{Z}_{i-1}, i_{\tilde{\xi}} \tilde{\Omega}_{1}(\tilde{z})=0, \eta_{Z^{*}}(\tilde{z})(\tilde{\xi})=1\right\}, \\
& \tilde{\operatorname{pr}}_{2}\left(\hat{W}_{i}\right)=Z_{i}=\left\{z \in Z_{i-1} / \exists \xi \in T_{z} Z_{i-1}, i_{\xi} \Omega_{L}(z)=0, \eta_{Z}(z)(\xi)=1\right\},
\end{aligned}
$$

for all $i \geq 2$. Moreover, one can construct the section $\beta$ of $\operatorname{Leg}_{f}: Z_{f} \rightarrow \tilde{Z}_{f}$ and the submanifold $\beta\left(\tilde{Z}_{f}\right)$ of $Z_{f}$ where a solution of the Euler-Lagrange equations exists.

The constraint algorithms using equations (4) and (11) and the construction of the corresponding constraint submanifolds $Z_{i}$ and $\tilde{Z}_{i}$ and of the submanifold $\beta\left(\tilde{Z}_{f}\right)$ has been done in $[22]$ (see also $[6,26]$ ). We remark that, in this case, there exists a unique solution of the Euler-Lagrange equations on the submanifold $\beta\left(\tilde{Z}_{f}\right)$ (for more details, see [22]).

The previous approach to time-dependent mechanics was also proposed in a recent contribution by Cortés, Martínez and Cantrijn [7]. 


\section{Appendices}

A. Projectable connections. A connection $\Gamma$ in the fibration $\pi_{X Y}: Y \rightarrow X$ is given by a horizontal distribution $\mathbf{H}$ which is complementary to the vertical one $V \pi_{X Y}$, that is,

$$
T Y=\mathbf{H} \oplus V \pi_{X Y}
$$

Associated to the connection there exists a horizontal projector $\mathbf{h}: T Y \rightarrow \mathbf{H}$ defined in the obvious manner.

If $\left(x^{\mu}, y^{i}\right)$ are fibered coordinates, then $\mathbf{H}$ is locally spanned by the local vector fields

$$
\left(\frac{\partial}{\partial x^{\mu}}\right)^{h}=\frac{\partial}{\partial x^{\mu}}+\Gamma_{\mu}^{i}(x, y) \frac{\partial}{\partial y^{i}}
$$

$\left(\partial / \partial x^{\mu}\right)^{h}$ is called the horizontal lift of $\partial / \partial x^{\mu}$, and $\Gamma_{\mu}^{i}$ are the Christoffel components of the connection.

Along the paper we repeatedly use the following construction.

Assume that $\pi_{X Z}: Z \rightarrow X$ and $\pi_{X Y}: Y \rightarrow X$ are two fibrations with the same base manifold $X$, and that $\Phi: Z \rightarrow Y$ is a surjective submersion (in other words, a fibration as well) preserving the fibrations, say, $\pi_{X Y} \circ \Phi=\pi_{X Z}$.

Let $\Gamma$ be a connection in $\pi_{X Z}: Z \rightarrow X$ with horizontal projector $\mathbf{h}$.

Definition A.1. $\Gamma$ is said to be projectable if $T \Phi(z)\left(\mathbf{H}_{z}\right)=T \Phi\left(z^{\prime}\right)\left(\mathbf{H}_{z^{\prime}}\right)$, for all $z, z^{\prime} \in Z$ in the same fiber of $\Phi$.

If $\Gamma$ is projectable, then we define a connection $\Gamma^{\prime}$ in the fibration $\pi_{X Y}: Y \rightarrow X$ as follows: The horizontal subspace at $y \in Y$ is given by

$$
\overline{\mathbf{H}}_{y}=T \Phi(z)\left(\mathbf{H}_{z}\right),
$$

for an arbitrary $z$ in the fiber of $\Phi$ over $y$. It is routine to prove that $\overline{\mathbf{H}}$ defines a horizontal distribution in the fibration $\pi_{X Y}: Y \rightarrow X$.

We can choose fibered coordinates $\left(x^{\mu}, y^{i}, z^{a}\right)$ on $Z$ such that $\left(x^{\mu}, y^{i}\right)$ are fibered coordinates on $Y$. The Christoffel components of $\Gamma$ are obtained by computing the horizontal lift

$$
\left(\frac{\partial}{\partial x^{\mu}}\right)^{h}=\frac{\partial}{\partial x^{\mu}}+\Gamma_{\mu}^{i}(x, y, z) \frac{\partial}{\partial y^{i}}+\Gamma_{\mu}^{a}(x, y, z) \frac{\partial}{\partial z^{a}} .
$$

A simple computation shows that $\Gamma$ is projectable if and only if the Christoffel components $\Gamma_{\mu}^{i}$ are constant along the fibers of $\Phi$, say $\Gamma_{\mu}^{i}=\Gamma_{\mu}^{i}(x, y)$. In this case, the horizontal lift of $\partial / \partial x^{\mu}$ with respect to $\Gamma^{\prime}$ is just

$$
\left(\frac{\partial}{\partial x^{\mu}}\right)^{h}=\frac{\partial}{\partial x^{\mu}}+\Gamma_{\mu}^{i}(x, y) \frac{\partial}{\partial y^{i}}
$$

As an exercise, the reader can easily check that, conversely, given a connection $\Gamma^{\prime}$ in the fibration $\pi_{X Y}: Y \rightarrow X$ and a surjective submersion $\Phi: Z \rightarrow Y$ preserving the fibrations, one can construct a connection $\Gamma$ in the fibration $\pi_{X Z}: Z \rightarrow X$ which projects onto $\Gamma^{\prime}$. 
B. Semiholonomic connections. Let $\pi_{X Y}: Y \rightarrow X$ be a fibration and $\pi_{X Z}: Z \rightarrow$ $X$ its 1 -jet prolongation, that is, $Z=J^{1} \pi_{X Y}$. Assume that $X$ is orientable with volume form $\eta$.

Definition B.2. A connection $\Gamma$ in the fibration $\pi_{X Z}: Z \rightarrow X$ is said to be semiholonomic if

$$
S_{\eta}(\mathbf{h}, \ldots, \mathbf{h})=0,
$$

where $\mathbf{h}$ is the horizontal projector of $\Gamma$. If (23) holds at a point $z \in Z$, then $\Gamma$ is said to be semiholonomic at $z$.

Assume that

$$
\mathbf{h}\left(\frac{\partial}{\partial x^{\mu}}\right)=\frac{\partial}{\partial x^{\mu}}+\Gamma_{\mu}^{i} \frac{\partial}{\partial y^{i}}+\Gamma_{\mu \nu}^{i} \frac{\partial}{\partial z_{\nu}^{i}}
$$

in fibered induced coordinates. Then $\Gamma$ is semiholonomic if and only if $\Gamma_{\mu}^{i}=z_{\mu}^{i}$.

C. Connections on submanifolds. The notion of connection in a fibration admits a useful generalization to submanifolds of the total space.

Let $\pi_{X Y}: Y \rightarrow X$ be a fibration and $P$ a submanifold of $Y$.

Definition C.1. A connection in $\pi_{X Y}: Y \rightarrow X$ along the submanifold $P$ consists of a family of linear mappings

$$
\mathbf{h}_{y}: T_{y} Y \rightarrow T_{y} P
$$

for all $y \in P$, satisfying

$$
\mathbf{h}_{y}^{2}=\mathbf{h}_{y}, \quad \operatorname{ker} \mathbf{h}_{y}=\left(V \pi_{X Y}\right)_{y},
$$

for all $y \in P$. The connection is said to be differentiable (flat) if the $n$-dimensional distribution $\operatorname{Im} \mathbf{h} \subset T P$ is smooth (integrable), where $n=\operatorname{dim} X$.

We have the following.

Proposition C.2. Let $\mathbf{h}$ be a connection in $\pi_{X Y}: Y \rightarrow$ Xalong a submanifold $P$ of Y. Then:

(1) $\pi_{X Y}(P)$ is an open subset of $X$.

(2) $\left(\pi_{X Y}\right)_{\left.\right|_{P}}: P \rightarrow \pi_{X Y}(P)$ is a fibration.

(3) The 1-jet prolongation $J^{1}\left(\pi_{X Y}\right)_{\left.\right|_{P}}$ is a submanifold of $Z$.

(4) There exists an induced true connection $\Gamma_{P}$ in the fibration $\left(\pi_{X Y}\right)_{\left.\right|_{P}}: P \rightarrow \pi_{X Y}(P)$ with the same horizontal subspaces.

(5) $\Gamma_{P}$ is flat if and only if $\mathbf{h}$ is flat.

Proof. (1) and (2). First of all, we shall prove that $\left(\pi_{X Y}\right)_{\left.\right|_{P}}: P \rightarrow X$ is a submersion. Let $y \in P$ such that $\pi_{X Y}(y)=x \in X$. We define a linear mapping

$$
\mathcal{A}(y): T_{x} X \rightarrow T_{y} P
$$

as follows:

$$
\mathcal{A}(y)(U)=\mathbf{h}_{y}(\bar{U})
$$


where $\bar{U} \in T_{y} Y$ and $T \pi_{X Y}(\bar{U})=U$. The mapping $\mathcal{A}(y)$ is well-defined since if $\bar{U}^{\prime}$ is another tangent vector in $T_{y} Y$ satisfying $T \pi_{X Y}\left(\bar{U}^{\prime}\right)=U$, then $\bar{U}-\bar{U}^{\prime} \in\left(V \pi_{X Y}\right)_{y}$, and therefore $\mathbf{h}_{y}\left(\bar{U}^{\prime}\right)=\mathbf{h}_{y}(\bar{U})$.

In addition, $\mathcal{A}(y)$ is injective. In fact, if $U \in T_{x} X$ is such that $\mathcal{A}(y)(U)=0$, then $\mathbf{h}_{y}(\bar{U})=0$, that implies $\bar{U} \in\left(V \pi_{X Y}\right)_{y}$, and therefore $U=T \pi_{X Y}(\bar{U})=0$.

Finally, $\mathcal{A}(y)$ is a section of $T \pi_{X Y}(y): T_{y} P \rightarrow T_{x} X$. Indeed, take $U \in T_{x} X$; we have $\mathcal{A}(y)\left(T \pi_{X Y}(\mathcal{A}(y)(U))\right)=\mathbf{h}_{y}(\mathcal{A}(y)(U))=\mathbf{h}_{y}^{2}(\bar{U})=\mathbf{h}_{y}(\bar{U})=\mathcal{A}(y)(U)$. Thus, we have proved that $T \pi_{X Y} \circ \mathcal{A}(y)=\operatorname{Id}_{T_{x} X}$. This shows that $\left(\pi_{X Y}\right)_{\left.\right|_{P}}: P \rightarrow X$ is a submersion.

Therefore, $\pi_{X Y}(P)$ is an open submanifold of $X$, and $\left(\pi_{X Y}\right)_{\left.\right|_{P}}: P \rightarrow \pi_{X Y}(P)$ is a fibration.

(3) is obvious.

(4) The induced connection $\Gamma_{P}$ is defined by restricting the horizontal subspaces of $\mathbf{h}$, that is,

$$
\mathbf{h}_{y}^{\prime}=\left(\mathbf{h}_{y}\right)_{\mid T_{y} P}, \quad \text { for all } y \in P .
$$

Since $\operatorname{Im} \mathbf{h}^{\prime}=\operatorname{Im} \mathbf{h}$ then (5) follows.

\section{References}

[1] C. Batle, J. Gomis and J. M. Pons, Hamiltonian and Lagrangian constraints of the bosonic string, Physical Review D 34 (1986), 2430-2432.

[2] E. Binz, M. de León, D. Martín de Diego and D. Socolescu, Nonholonomic constraints in classical field theories, Rep. Math. Phys. 49 (2002), 151-166.

[3] E. Binz, J. Śniatycki and H. Fischer, Geometry of Classical Fields, North-Holland Math. Stud. 154, North-Holland, Amsterdam, 1988.

[4] F. Cantrijn, A. Ibort and M. de León, Hamiltonian structures on multisymplectic manifolds, Rend. Sem. Mat. Univ. Pol. Torino 54 (1996), 225-236.

[5] F. Cantrijn, A. Ibort and M. de León, On the geometry of multisymplectic manifolds, J. Austral. Math. Soc. (Series A) 66 (1999), 303-330.

[6] D. Chinea, M. de León and J. C. Marrero, The constraint algorithm for time-dependent lagrangians, J. Math. Phys. 35 (1994), 3410-3447.

[7] J. Cortés, S. Martínez and F. Cantrijn, Skinner-Rusk approach to time-dependent mechanics, Phys. Lett. A 300 (2002), 250-258.

[8] A. Echeverría-Enríquez, M. C. Muñoz-Lecanda and N. Román-Roy, Multivector fields and connections. Setting Lagrangian equations for field theories, J. Math. Phys. 39 (1998), 4578-4603.

[9] A. Echeverría-Enríquez, M. C. Muñoz-Lecanda and N. Román-Roy, Multivector field formulation of Hamiltonian field theories: equations and symmetries, J. Phys. A: Math. Gen. 32 (1999), 8461-8484.

[10] A. Echeverría-Enríquez, M. C. Muñoz-Lecanda and N. Román-Roy, On the multimomentum bundles and the Legendre maps in field theories, Rep. Math. Phys. 45 (2000) 85-105.

[11] A. Echeverría-Enríquez, M. C. Muñoz-Lecanda and N. Román-Roy, Geometry of multisymplectic Hamiltonian first-order field theories, J. Math. Phys. 41 (2000), 7402-7444.

[12] P. L. García-Pérez and A. Pérez-Rendón, Symplectic approach to the theory of quantized fields, I, Comm. Math. Phys. 13 (1969), 24-44. 
[13] P. L. García-Pérez and A. Pérez-Rendón, Symplectic approach to the theory of quantized fields, II, Archive Rat. Mech. Anal. 43 (1971), 101-124.

[14] H. Goldschmidt and Sh. Sternberg, The Hamilton-Cartan formalism in the calculus of variations, Ann. Inst. Fourier (Grenoble) 23 (1975), 203-267.

[15] M. J. Gotay and J. Nester, Presymplectic Lagrangian systems I: the constraint algorithm and the equivalence theorem, Ann. Inst. Henri Poincaré A 30 (1978), 129-142.

[16] M. J. Gotay and J. Nester, Presymplectic Lagrangian systems II: the second order differential equation problem, Ann. Inst. Henri Poincaré A 32 (1980), 1-13.

[17] M. J. Gotay, J. Isenberg and J. E. Marsden, Momentum maps and classical relativistic fields, Part I: Covariant field theory, MSRI preprint (1997).

[18] M. J. Gotay, J. Isenberg and J. E. Marsden, Momentum maps and classical relativistic fields, Part II: Canonical analysis of field theories, MSRI preprint (1999).

[19] J. Kijowski and W. Tulczyjew, A Symplectic Framework for Field Theories, Lecture Notes in Physics 107, Springer, New York, 1979.

[20] M. McLean, M. de León, L. K. Norris, A. M. Rey and M. Salgado, Geometric Structures in Field Theory, preprint (2001).

[21] M. de León and J. C. Marrero, Constrained time-dependent Lagrangian systems and Lagrangian submanifolds, J. Math. Phys. 34 (1993), 622-644.

[22] M. de León, J. Marín-Solano and J. C. Marrero, The constraint algorithm in the jet formalism, Diff. Geom. Appl. 6 (1996), 275-300.

[23] M. de León, J. C. Marrero and J. Marín, A geometrical approach to Classical Field Theories: a constraint algorithm for singular theories, in: New Developments in Differential Geometry (Debrecen, 1994), Math. Appl. 350, Kluwer, Dordrecht, 1996, 291-312.

[24] M. de León, J. C. Marrero and J. Marín, Ehresmann connections in Classical Field Theories, in: Differential Geometry and its Applications (Granada, 1994), Anales de Física, Monografías, 2, 1995, 73-89.

[25] M. de León, D. Martín de Diego and A. Santamaría, Symmetries in classical field theories, in preparation.

[26] M. de León, J. Marín-Solano, J. C. Marrero, M. Muñoz-Lecanda and N. Román-Roy, Singular lagrangian systems on jet bundles, Fortschritte für Physik 50 (2002), 105-169.

[27] G. Martin, Dynamical structures for k-vector fields, Int. J. Theor. Phys. 27 (1988), 571585 .

[28] G. Martin, A Darboux theorem for multisymplectic manifolds, Letters Math. Phys. 16 (1988), 133-138.

[29] C. Paufler and H. Roemer, Geometry of Hamiltonian n-vector fields in multisymplectic field theory, J. Geom. Phys. 44 (2002), 52-69.

[30] C. Paufler and H. Roemer, De Donder-Weyl equations and multisymplectic geometry, Rep. Math. Phys. 49 (2002), 325-334.

[31] G. Sardanashvily, Gauge Theory in Jet Manifolds, Hadronic Press, Palm Harbour, Florida, 1993.

[32] G. Sardanashvily, Generalized Hamiltonian Formalism for Field Theory. Constraint Systems, World Scientific, Singapore, 1995.

[33] R. Skinner and R. Rusk, Generalized Hamiltonian dynamics I. Formulation on $T^{*} Q \oplus T Q$, J. Math. Phys. 24 (1983), 2589-2594.

[34] R. Skinner and R. Rusk, Generalized Hamiltonian dynamics. II. Gauge transformations, J. Math. Phys. 24 (1983), 2595-2601. 